\title{
Wireless-Sensor Networks in Space Technology Demonstration on ISS
}

\author{
Dr. H. J. Beestermöller ${ }^{1}$, J. Sebald, \\ M.-C. Sinnreich ${ }^{1}$, H.-J. Borchers ${ }^{1}$, M. Schneider ${ }^{1}, H$. Luttmann ${ }^{1}$, V. Schmid ${ }^{2}$ \\ ${ }^{1}$ Airbus D\&S, Bremen, Germany, \\ Hans-Joerg.Beestermoeller@airbus.com \\ Johannes.Sebald@airbus.com \\ ${ }^{2}$ Deutsches Zentrum für Luft- und Raumfahrt (DLR), Bonn, Germany \\ Volker.Schmid@DLR.de
}

\begin{abstract}
:
Wireless Sensor Networks (WSN) consist of distributed sensors enabling monitoring of physical or environmental conditions like temperature, pressure, humidity, accelerations etc. When a sensor node includes a micro-controller, the acquired data can also be pre-processed e.g. to reduce the amount of data to be transmitted. The network can be organized in various topology types. Short-range networks based on wireless networking architectures enable power efficient network architectures and have enabled new wireless machine-to-machine applications.
\end{abstract}

For safety critical space applications e.g. launchers, satellites or human space flight additionally criteria like mass, power demand and robustness against harsh environment ought to be considered. Wireless systems evolved in the last years exactly in this direction and thus they are recognised even by the space agencies as key for future applications.

Focus of this article is the engineering approaches currently discussed. It presumes the availability of adequate sensing systems or at least with minor need for adaptations. Some more insight is given with regards to an In-orbit Demonstration (IOD) of a Wireless-Sensor Network, which was tested on the ISS.

Key words: Wireless-Sensor Networks, Reliable-Sensor Networks, Space Applications, International Space Stations, Microelectromechanical systems.

\section{Wired Sensors versus Wireless}

Electrical systems in space require a lot of sensors to be properly operated. As an example the current Ariane 5 uses over 500 wired sensors for functional and telemetry purposes. The installation and possible change of configuration requires a significant effort in documentation, installation and test. The related wiring, connectors, brackets and other mechanical parts to integrate the harness are summing up to a mass of hundreds of $\mathrm{kg}$. The substitution of wired sensors by wireless sensors offers the opportunity to save mass and documentation/integration effort in the future.

Wireless sensor networks not only have to communicate without wires but need to be supplied autonomously with electrical energy as well. To supply this energy, batteries can be used. However energy harvesting methods to convert available non-electrical energy are under investigation. There are three main energy sources available in space: Mechanical Energy converted by Piezo-Elements, Electromagnetic Energy converted by appropriate circuits or by solar cells (optical range) and Thermal Energy converted by Thermoelectric Devices.

\section{Wireless Technologies}

Wireless communication can be established via different technologies. Narrow-Band RF communication like WLAN (IEEE 802.11 [1]) or Bluetooth (IEEE 802.15.1 [2]) is used nearly everywhere today. However theses kinds of Narrow-Band communication are sensitive to interference and have some EMC issues. Therefore Ultra-wideband (UWB) RF Communication (IEEE 802.15.4 [3]) has recently gained attention.

Another technology insensitive to interference and EMC is optical communication in the visible or infrared regime. 


\section{Down selection for Launchers}

The environment for wireless physical channels inside a Launcher is highly reflective as of multiple electrical conducting surfaces inside the launcher and ubiquitous usage of Multilayer Isolation. The operation has to be established in closed electromagnetic compartments.

In this environment Narrow-Band RF communication has some severe drawbacks due to destructive interference caused by multipath reflection inside the compartments.

The two other technologies UWB and optical communication are much more suitable to be operated under these conditions, as they are much less sensitive to multipath effects.

Feasibility tests [4] inside an electromagnetic flight representative Mock-Up of the Vehicle Equipment Bay (VEB) of an Ariane 5 Launcher have shown that these two technologies UWB and IR are able to transmit the required sensor data inside the launcher in Line of Sight (LOS) and Non Line of Sight (NLOS) arrangements.

Therefore UWB and IR technologies are under further investigation for use in Launcher wireless sensor networks.

\section{Down selection for use on ISS}

When looking at a possible use of a wireless sensor network on the ISS a maximum range of $50 \mathrm{~m} \times 60 \mathrm{~m}$ has to be considered, presuming the application within the pressurized modules. Though, considering a minimised number of base stations, the network should support a multi-hop network topology allowing forwarding data from sensor nodes not having direct contact to a base station. Such kinds of networks are supported by systems based on the IEEE 802.15.4 [3] standard. It operates on one of three possible unlicensed frequency bands, but only the one at $2.4 \mathrm{GHz}$ is allowed worldwide and though preferred for application on ISS. On the other hand Bluetooth and WLAN are operating in the same frequency range. Interferences with mobile devices, e.g. tablet PCs or laptops, may occur and disturb the operation of a wireless-sensor network. This becomes even more important, because the requirements for electromagnetic compatibility, which are applicable, are limiting the allowed emitting power significantly. This fits perfectly concerning the power demand for the sensor nodes, but makes it even worse wrt the stability of the communication.

As an alternative an infra-red (IR) based communication system could be used. Concerning the mandatory regulations wrt the safety of the station and especially for the crew on-board the ISS, such systems have clear advantages. IR seems furthermore attractive, because there is only little equipment using this technology. On the other hand, the configuration of laboratory equipments, tools etc. are permanently changed and thus the operational environment is not stable. Fig. 1 is depicting as a typical environment the interior of the Columbus module, which is the European laboratory of the ISS. As an example Even the astronauts are moving through the IR-beams and in comparison with RF based systems a IR based communication is though expected to be disturbed more frequently.

A more detailed trade-off between several systems, taking into account also availability of components, maturity of the technology etc., carried out, that a RF based system is the best option.

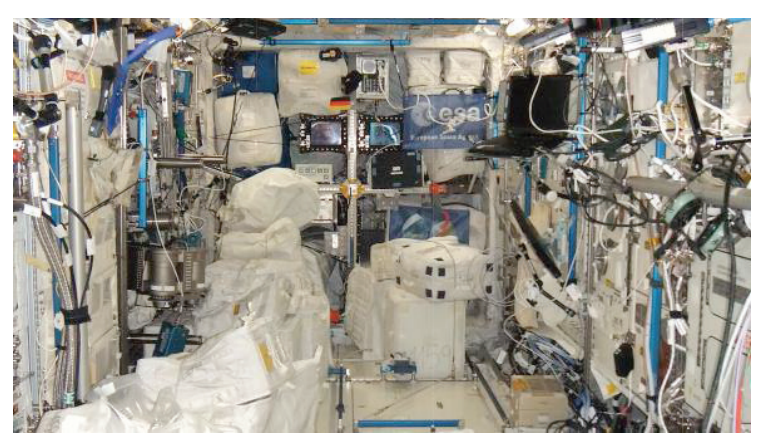

Fig. 1: Interior of Columbus (Courtesy NASA)

\section{In-orbit Demonstration of RF Technology}

In preparation of the mission of Alexander Gerst, which took place in 2014, the European Space Agency (ESA) and the German national agency DLR offered to industry the opportunity for doing technology demonstration on the International Space Station. This offer was coming along with a strong requirement on time for implementation, i.e. 8 months between start of activity and delivery for launch.

However, this was an exceptional chance to demonstrate the technology readiness for use in space applications and furthermore in the 'real' environment. To cope with the schedule the decision was to use widely commercial of the shelf components which could be modified for use on the ISS. Finally it was decided to use four sensor nodes in the Columbus module only. Each of the nodes was equipped with environmental sensors for air humidity, air pressure, temperature, 2-axis accelerometer and ambient light. The latter was selected to obtain a clear picture of the actual situation onboard. With the use of Microelectromechanical (MEMS) devices in combination with the low power communication the thesis was that it 
should be possible to operate the system without using any batteries,

The base station of the network, as shown in Fig. 2 was placed at on the front panel of an experiment insert and partially covered by hoses. For a RF-based system this is not ideal. Furthermore, the NASA Frequency management group, responsible entity to endorse the use of RF systems on the ISS, required the limitation of the power to $1 \mathrm{~mW}$.

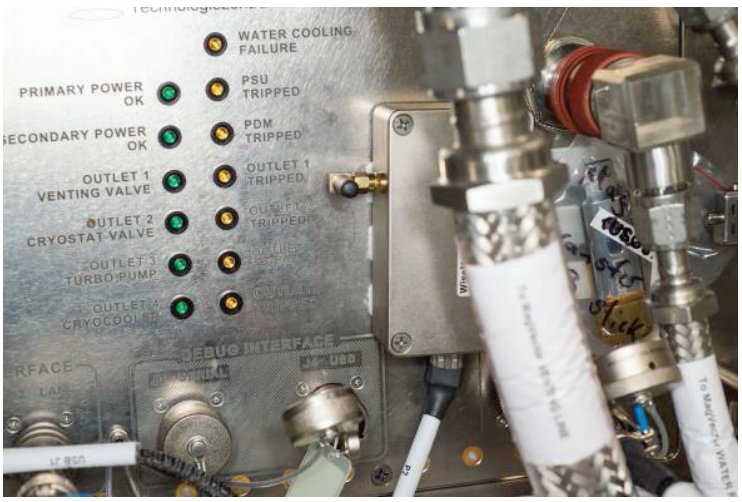

Fig. 2: WiSe-Net Base-station (Courtesy NASA)

Even the selection of the locations for the sensor modes was done to have different grades of influence on operational stability and of course concerning the light sources. Fig 3 is depicting one of the nodes, which is, compared to the others, in a quite good location.

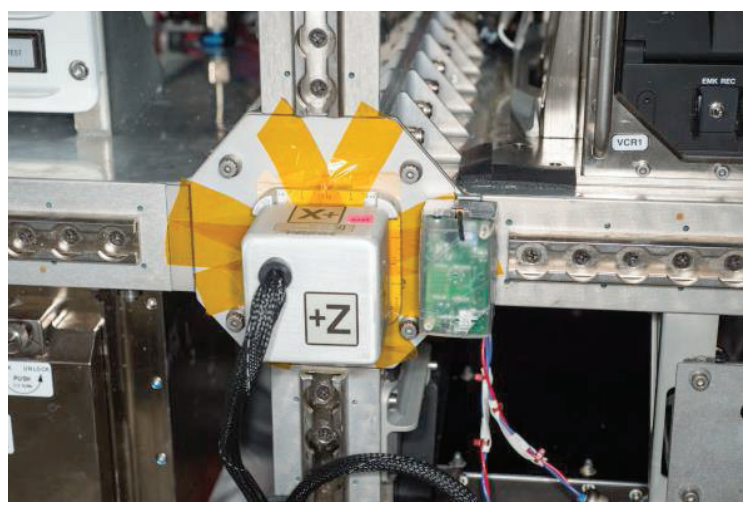

Fig. 3: One WiSe-Net Sensor Node with transparent housing (Courtesy NASA)

WiSe-Net was installed at New Year's Eve 2014 and was activated directly afterwards. The first run of the technology demonstration was very successful. The evaluation of the data showed, that even though having a difficult environment, the RF communication was extremely stable. Of course there were several data packet repetitions reported, especially from those nodes shielded somehow by metallic items, but there was nearly no packet loss. Even more, the ability of the system to automatically establish a multi-hop network was not necessary. Each of the nodes had a direct link to the base-station. Concerning the use of ambient light as a power source, the data shows surprisingly a better situation as expected. Certainly there are some limitations concerning the usability of photovoltaic, but at least for the hardware used the source would be enough to avoid using batteries.

The quite successful demonstration led to the planning for a second run. Objective is now to spread the nodes over several modules of the ISS, as shown in Fig. 4.

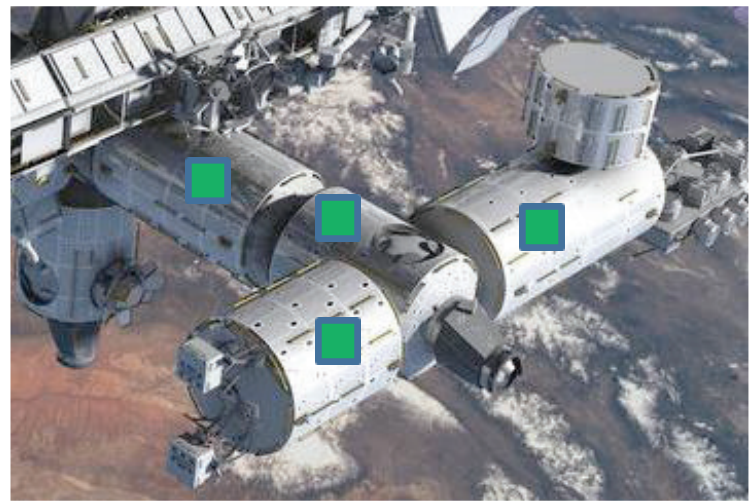

Fig.4: $\quad$ Distributed WiSe-Net Sensor Nodse

In this scenario it is expected, that the network will use the packet forwarding to the basestation, which will remain in the Columbus module.

\section{In-orbit Demonstration of IR Technology}

In parallel to the preparation of the second run of the RF-based system, an infra-red based wireless sensor network is under development, that will be tested inside the ISS using the hardware infrastructure already used for the first WiSe-Net campaign as far as possible, to reduce the development effort to the necessary minimum.

The same set of sensors will be used for the wireless sensor nodes and they will be placed in the same positions as the sensors of the first WiSe-Net campaign to be able to compare the results with the already acquired data from the RF based system.

\section{Conclusion and Outlook}

The first WiSe-Net campaign has shown the function of a COTS based Narrow-Band RF wireless sensor network inside the ISS and has opened further possibilities to evaluate wireless sensor network technologies in space.

The next steps will be an extension of the original WiSe-Net and an implementation of a comparable optical (IR) sensor network.

An implementation of an UWB based sensor network in the next years on the ISS is currently under investigation. 
As the wireless sensor network technology has been shown to be usable in the ISS environment different applications are in reach for the future:

- WiSe-Net Gas-detection

The continuous measurement of gas concentrations of different substances (Ammonia, Carbon Dioxide, etc.) is interesting to improve the operation of the ISS. Related sensors can be developed and used in the ISS.

- BAN

Body area networks (BAN) for health parameter surveillance of astronauts, especially during Extravehicular activities (EVA) may be significantly improved by use of wireless technologies. As UWB technologies allow for localization of sensors, this may also be used in advantage during EVA to get the position of astronauts relative to each other and to the ISS.

Launcher wired sensor networks have a high potential for improvement by using wireless sensor technologies. Especially UWB and IR Technologies have been identified as possible candidates for establishment of wireless sensor sub-networks.

In the frame of the ESA Future Launchers Preparatory Programme (FLPP), the project "Reliable Sensor Network" (RSN) has been started in 2014 to develop a heterogeneous Telemetry-Network for Sensor data acquisition.

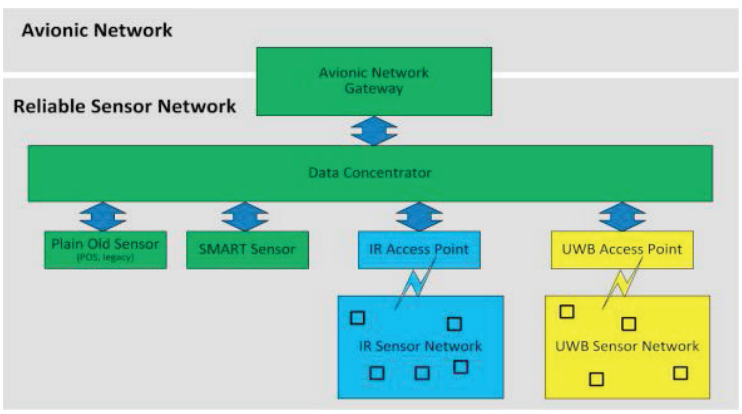

Fig.5: Reliable Sensor Network

Figure 5 shows the concept of RSN which consists of a Gateway to the Avionic Network, a logical layer called Data Concentrator, which is mainly consisting off a local data bus and different kind of sensor interfaces/access points, related to the following kind of sensors:

- Analog wired sensors currently in use on Ariane 5

- Digital (SMART) wired sensors for future application, linked via a serial bus like CAN, I2C etc.

- Wireless UWB sensors communicating via an UWB Access Point with the System
- Wireless IR sensors communicating via an IR Access Point with the System

The RSN heterogeneous architecture has the following benefits:

- Smooth technology transition (part by part implementation)

- Backward compatibility

- Smooth future development (extendibility)

As a layered, open architecture is envisaged the concept is optimized for:

- Availability of components (no single source)

- Obsolescence relaxation (fixed interfaces)

- Easy optimization during exploitation phase

In the frame of the RSN project an engineering model will be developed. This model will be characterized inside a flight representative Mock-Up of the Vehicle Equipment Bay (VEB) especially to show operation of the wireless subsystems in an electromagnetic representative environment.

\section{References}

[1] IEEE Std 802.11; Standard for Information technology, Part 11: Wireless LAN Medium Access Control (MAC) and Physical Layer (PHY) Specifications; 2012

[2] IEEE Std 802.15.1; Standard for Telecommunications and Information Exchange between Systems -LAN/MAN - Specific Requirements, Part 15:Wireless Medium Access Control (MAC) and Physical Layer (PHY) Specifications for Wireless Personal Area Networks (WPANS); 2002

[3] IEEE Std 802.15.4; Standard for Information technology, Part 15.4: Wireless Medium Access Control and Physical Layer Specifications for Low-Rate Wireless Personal Area Networks; 2006

[4] Feasibility Measurement: To be published 